PROCEEDINGS OF THE

AMERICAN MATHEMATICAL SOCIETY

Volume 125, Number 7, July 1997, Pages 2133-2139

S 0002-9939(97)03896-3

\title{
LOCAL DIRICHLET SPACES AS DE BRANGES-ROVNYAK SPACES
}

\author{
DONALD SARASON
}

(Communicated by Theodore W. Gamelin)

\begin{abstract}
The harmonically weighted Dirichlet spaces associated with unit point masses are shown to coincide with de Branges-Rovnyak spaces, with equality of norms. As a consequence it is shown that radial expansion operators act contractively in harmonically weighted Dirichlet spaces.
\end{abstract}

\section{INTRODUCTION}

The Dirichlet-type spaces of S. Richter, referred to here as harmonically weighted Dirichlet spaces, arose in the study of two-isometries [3]. The two-isometries form a natural class of operators whose most prominent member is the Dirichlet shift, the operator of multiplication by the independent variable on the classical Dirichlet space of the unit disk.

A harmonically weighted Dirichlet space is associated with each finite positive Borel measure on the unit circle; it consists of the holomorphic functions in the disk whose derivatives are square integrable when weighted against the Poisson integral of the measure. In case the measure is normalized Lebesgue measure, it is the classical Dirichlet space, the space on which the Dirichlet shift acts. These spaces have received a penetrating analysis in a series of papers of Richter and C. Sundberg [4], [5], [6]. It is the purpose of this paper and a successor [8] to delve further into the case where the measure in question is a finite sum of atoms. The present paper pertains to the simplest subcase, that of a unit point mass.

For $\mu$ a finite positive measure on $\partial \mathbf{D}$ (the unit circle) and $f$ a holomorphic function in $\mathbf{D}$ (the unit disk), the Dirichlet integral of $f$ with respect to $\mu$ is defined by

$$
D_{\mu}(f)=\int_{\mathbf{D}}\left|f^{\prime}\right|^{2} P \mu d A,
$$

where $P \mu$ is the Poisson integral of $\mu$, and $A$ denotes area measure on $\mathbf{D}$, normalized to have unit total mass. In case $\mu$ is $\delta_{\lambda}$, the unit point mass at the point $\lambda$ of $\partial \mathbf{D}$, one writes $D_{\lambda}(f)$ for $D_{\mu}(f)$, and refers to $D_{\lambda}(f)$ as the local Dirichlet integral of $f$ at $\lambda$. A straightforward application of Fubini's theorem gives the equality

$$
D_{\mu}(f)=\int_{\partial \mathbf{D}} D_{\lambda}(f) d \mu(\lambda) .
$$

Received by the editors February 13, 1996.

1991 Mathematics Subject Classification. Primary 46E20.

(C)1997 American Mathematical Society 
The harmonically weighted Dirichlet space $D(\mu)$ consists of those $f$ for which $D_{\mu}(f)<\infty$. It turns out to be contained in the Hardy space $H^{2}$, and it is a Hilbert space under the norm $\|\cdot\|_{\mu}$ defined by

$$
\|f\|_{\mu}^{2}=\|f\|_{2}^{2}+D_{\mu}(f),
$$

where $\|\cdot\|_{2}$ denotes the norm in $H^{2}$. The harmonically weighted Dirichlet spaces corresponding to unit point masses are the local Dirichlet spaces of the paper's title. (The harmonically weighted Dirichlet space associated with the zero measure is defined to be just $H^{2}$.)

Let $S$ denote the shift operator on $H^{2}$ (the operator of multiplication by the independent variable). A basic result of Richter and Sundberg [4, Proposition 2.2] states that, for $\lambda$ in $\partial \mathbf{D}$, the function $f$ belongs to $D\left(\delta_{\lambda}\right)$ if and only if it differs by a constant from a function in the range of $S-\lambda I$ :

$$
f=c+(S-\lambda I) g
$$

in that case $D_{\lambda}(f)=\|g\|_{2}^{2}$. The constant $c$ is the nontangential limit of $f$ at $\lambda$, so the last equality can be written

$$
D_{\lambda}(f)=\frac{1}{2 \pi} \int_{\partial \mathbf{D}}\left|\frac{f(z)-f(\lambda)}{z-\lambda}\right|^{2} d m(z)
$$

where $m$ denotes normalized Lebesgue measure on $\partial \mathbf{D}$. An alternative and somewhat smoother proof of this result is presented in Section 2.

In Section 3 it is shown that the local Dirichlet spaces $D\left(\delta_{\lambda}\right)(\lambda \in \partial \mathbf{D})$, besides being the simplest harmonically weighted Dirichlet spaces, belong to another class of spaces that have received recent attention, the de Branges-Rovnyak spaces. That the spaces $D\left(\delta_{\lambda}\right)$ can be identified with certain de Branges-Rovnyak spaces, up to equivalence of norms, was noted already in [4, Proposition 2.4]. The novelty here is that the identification is norm preserving.

For $0<r<1$, let $C_{r}$ denote the composition operator induced by the function $r z:\left(C_{r} f\right)(z)=f(r z)$. Richter and Sundberg [4, Theorem 5.2] have proved that if $f$ is in $D(\mu)$, then $D_{\mu}\left(C_{r} f\right) \leq 4 D_{\mu}(f)$. As an application of the result in Section 3, it will be shown in Section 4 that the constant 4 in this inequality can be replaced by 1 . A consequence concerning the boundary behavior of functions in $D(\mu)$ will be indicated. (Sundberg has proved the same result using different methods. To my knowledge his proof is unpublished.)

\section{The local Dirichlet integral as a Boundary integral}

For $\lambda$ in $\partial \mathbf{D}$, let $\mathcal{M}(S-\lambda I)$ denote the range of the operator $S-\lambda I$, endowed with the range norm (the norm that makes $S-\lambda I$ an isometry of $H^{2}$ onto its range). Let $A^{2}\left(P \delta_{\lambda}\right)$ denote the space of holomorphic functions in $\mathbf{D}$ that are in $L^{2}\left(P \delta_{\lambda} d A\right)$, with the norm inherited from $L^{2}\left(P \delta_{\lambda} d A\right)$.

Proposition 1. The operator of differentiation is an isometry of $\mathcal{M}(S-\lambda I)$ onto $A^{2}\left(P \delta_{\lambda}\right)$.

This proposition gives the result of Richter and Sundberg, mentioned in Section 1 , that a function is in $D\left(\delta_{\lambda}\right)$ if and only if it differs by a constant from a function in $\mathcal{M}(S-\lambda I)$, as well as their expression for $D_{\lambda}$ as a boundary integral.

The obvious change of variables enables one to reduce Proposition 1 to the case $\lambda=1$. We let $\mathcal{M}^{\prime}(S-I)$ denote the space of derivatives of functions in $\mathcal{M}(S-I)$, 
with the norm inherited from $\mathcal{M}(S-I)$. The spaces $\mathcal{M}^{\prime}(S-I)$ and $A^{2}\left(P \delta_{1}\right)$ are both reproducing kernel Hilbert spaces. The proposition is just the statement that these two spaces are identical (as Hilbert spaces). To establish this it will be enough to show that their kernel functions coincide.

We first determine the kernel function of $\mathcal{M}^{\prime}(S-I)$. As is well known, the kernel function for the space $H^{2}$ is $(1-\bar{w} z)^{-1}$. The space $\mathcal{M}(S-I)$ is the isometric image of $H^{2}$ under the operator of multiplication by the function $z-1$, from which one concludes that its kernel function is the function

$$
\frac{(\bar{w}-1)(z-1)}{1-\bar{w} z} .
$$

It follows that the kernel function in $\mathcal{M}(S-I)$ for evaluation of the derivative is

$$
\frac{\partial}{\partial \bar{w}}\left[\frac{(\bar{w}-1)(z-1)}{1-\bar{w} z}\right]
$$

in other words, for fixed $w$ in $\mathbf{D}$, the inner product in $\mathcal{M}(S-I)$ of a function $f$ with the preceding function (of $z$ ) equals $f^{\prime}(w)$. Because differentiation is an isometry of $\mathcal{M}(S-I)$ onto $\mathcal{M}^{\prime}(S-I)$, one concludes that the kernel function for $\mathcal{M}^{\prime}(S-I)$ is the function

$$
\frac{\partial^{2}}{\partial z \partial \bar{w}}\left[\frac{(\bar{w}-1)(z-1)}{1-\bar{w} z}\right] .
$$

After a calculation one finds that the kernel function for $\mathcal{M}^{\prime}(S-I)$ is the function

$$
\frac{2(1-\bar{w})(1-z)}{(1-\bar{w} z)^{3}} \text {. }
$$

To find the kernel function for the space $A^{2}\left(P \delta_{1}\right)$, we first let $\rho(z)=1-|z|^{2}$ and consider the space $A^{2}(\rho)$ of holomorphic functions in $\mathbf{D}$ that are in $L^{2}(\rho d A)$. By standard reasoning one sees that the powers of $z$ form an orthogonal basis for $A^{2}(\rho)$, the square of the norm of $z^{n}$ equaling $1 /(n+1)(n+2)$. It follows that the kernel function for $A^{2}(\rho)$ is the function

$$
\sum_{n=0}^{\infty}(n+1)(n+2) \bar{w}^{n} z^{n}=\frac{2}{(1-\bar{w} z)^{3}} .
$$

Because $P \delta_{1}(z)=\left(1-|z|^{2}\right) /|1-z|^{2}$, the space $A^{2}\left(P \delta_{1}\right)$ is the isometric image of $A^{2}(\rho)$ under the operator of multiplication by the function $1-z$. The kernel function for $A^{2}\left(P \delta_{1}\right)$ is therefore the function

$$
\frac{2(1-\bar{w})(1-z)}{(1-\bar{w} z)^{3}} .
$$

This concludes the proof of Proposition 1.

\section{Local Dirichlet spaces as de Branges-Rovnyak spaces}

The Toeplitz operator on $H^{2}$ whose symbol is the function $\phi$ in $L^{\infty}$ of $\partial \mathbf{D}$ will be denoted, as usual, by $T_{\phi}$. For $b$ a function in the unit ball of $H^{\infty}$, the de BrangesRovnyak space $\mathcal{H}(b)$ is the range of the operator $\left(I-T_{b} T_{\bar{b}}\right)^{\frac{1}{2}}$ with the range norm, which is denoted by $\|\cdot\|_{b}$. These spaces were first studied by L. de Branges and 
J. Rovnyak [1]; a recent account is [7]. The space $\mathcal{H}(b)$ is a reproducing kernel Hilbert space whose kernel function is

$$
\frac{1-\overline{b(w)} b(z)}{1-\bar{w} z}
$$

Let $w_{0}$ denote the smaller of the two solutions of the equation $w=(1-w)^{2}$ :

$$
w_{0}=\frac{3-\sqrt{5}}{2} .
$$

(The number $w_{0}$ is the reciprocal of the square of the golden mean.) For $\lambda$ in $\partial \mathbf{D}$, we define the function $b_{\lambda}$ by

$$
b_{\lambda}(z)=\frac{\left(1-w_{0}\right) \bar{\lambda} z}{1-w_{0} \bar{\lambda} z} .
$$

Proposition 2. $D\left(\delta_{\lambda}\right)=\mathcal{H}\left(b_{\lambda}\right)$, with equality of norms.

One way to prove this proposition, the way it was discovered, is to compute the kernel function of $D\left(\delta_{\lambda}\right)$ and observe that it equals the kernel function of $\mathcal{H}\left(b_{\lambda}\right)$. Once one knows the result, however, it is less cumbersome to work in the opposite direction. The argument uses a few facts about de Branges-Rovnyak spaces which can be found in [7].

The required facts are as follows. If the function $b$ in the unit ball of $H^{\infty}$ has the property that $1-|b|^{2}$ is log-integrable on $\partial \mathbf{D}$, then there is a unique outer function $a$ such that $a(0)>0$ and $|a|^{2}+|b|^{2}=1$ almost everywhere on $\partial \mathbf{D}$. Then the $H^{2}$ function $f$ belongs to $\mathcal{H}(b)$ if and only if there is an $H^{2}$-function $f^{+}$, necessarily unique, such that $T_{\bar{b}} f=T_{\bar{a}} f^{+}$. The square of the norm of $f$ in $\mathcal{H}(b)$ is the sum of the squares of the $H^{2}$ norms of $f$ and $f^{+}$:

$$
\|f\|_{b}^{2}=\|f\|_{2}^{2}+\left\|f^{+}\right\|_{2}^{2} .
$$

For $\lambda$ a point of $\partial \mathbf{D}$ and $|z|=1$, we have

$$
\begin{aligned}
1-\left|b_{\lambda}(z)\right|^{2} & =1-\frac{w_{0}}{\left|1-w_{0} \bar{\lambda} z\right|^{2}}=\frac{1+w_{0}^{2}-w_{0}-2 w_{0} \operatorname{Re} \bar{\lambda} z}{\left|1-w_{0} \bar{\lambda} z\right|^{2}} \\
& =\frac{2 w_{0} \operatorname{Re}(1-\bar{\lambda} z)}{\left|1-w_{0} \bar{\lambda} z\right|^{2}}=\frac{\left(1-w_{0}\right)^{2}|1-\bar{\lambda} z|^{2}}{\left|1-w_{0} \bar{\lambda} z\right|^{2}} .
\end{aligned}
$$

(The equality $w_{0}=\left(1-w_{0}\right)^{2}$ was used three times in the preceding calculation.) Letting $a_{\lambda}$ denote the function $a$ that arises when $b=b_{\lambda}$, we thus have the expression

$$
a_{\lambda}(z)=\frac{\left(1-w_{0}\right)(1-\bar{\lambda} z)}{\left(1-w_{0} \bar{\lambda} z\right)}
$$

We consider now a function $f$ in $H^{2}$ and use the criterion mentioned above to determine when $f$ is in $\mathcal{H}\left(b_{\lambda}\right)$. Thus, we want to determine when there is a function 
$f^{+}$in $H^{2}$ such that $T_{\bar{b}_{\lambda}} f=T_{\bar{a}_{\lambda}} f^{+}$. For $g$ any function in $H^{2}$, we have, for $|z|=1$,

$$
\begin{aligned}
\bar{b}_{\lambda} f-\bar{a}_{\lambda} g & =\frac{\left(1-w_{0}\right)}{1-w_{0} \lambda \bar{z}}[\lambda \bar{z} f-(1-\lambda \bar{z}) g] \\
& =\frac{\left(1-w_{0}\right) \lambda \bar{z}}{1-w_{0} \lambda \bar{z}}[f-\bar{\lambda}(z-\lambda) g] .
\end{aligned}
$$

The equality $T_{\bar{b}_{\lambda}} f=T_{\bar{a}_{\lambda}} g$ holds if and only if the preceding function is orthogonal to $H^{2}$, which happens if and only if $f-\bar{\lambda}(z-\lambda) g$ is constant. Conclusion: $f$ is in $\mathcal{H}\left(b_{\lambda}\right)$ if and only if it differs by a constant from a function in the range of $S-\lambda I$. Then $f^{+}$is the function in $H^{2}$ such that $f-(S-\lambda I) \bar{\lambda} f^{+}$is constant. From Proposition 1 we see that the spaces $D\left(\delta_{\lambda}\right)$ and $\mathcal{H}\left(b_{\lambda}\right)$ are equal, and that the equality $\left\|f^{+}\right\|_{2}^{2}=D_{\lambda}(f)$ holds for $f$ in $\mathcal{H}\left(b_{\lambda}\right)$. The last equality implies that the norms in $D\left(\delta_{\lambda}\right)$ and $\mathcal{H}\left(b_{\lambda}\right)$ are the same. This completes the proof of Proposition 2.

\section{Contractivity of EXPANSIONS}

Recall that, for $0<r<1$, the composition operator $C_{r}$ was defined in Section 1: $\left(C_{r} f\right)(z)=f(r z)$. One can think of $C_{r} f$ as being obtained from $f$ by expanding its domain. When convenient, we shall write $f_{r}$ in place of $C_{r} f$.

Proposition 3. For $\mu$ a finite positive measure on $\partial \mathbf{D}$ and $0<r<1$, the operator $C_{r}$ acts contractively in $D(\mu)$.

Because of (1), it will suffice to prove this for the case where $\mu$ is a unit point mass. The argument will be given for the case $\mu=\delta_{1}$, the more general case $\mu=\delta_{\lambda}$ being essentially the same. The identity of the spaces $D\left(\delta_{1}\right)$ and $\mathcal{H}\left(b_{1}\right)$ will be used. For simplicity we let $b=b_{1}$ and $a=a_{1}$ :

$$
b(z)=\frac{\left(1-w_{0}\right) z}{1-w_{0} z}, \quad a(z)=\frac{\left(1-w_{0}\right)(1-z)}{1-w_{0} z} .
$$

Proposition 2 and its proof show that, to prove Proposition 3, it will suffice to prove that $\left\|\left(C_{r} f\right)^{+}\right\|_{2} \leq\left\|f^{+}\right\|_{2}$ for all $f$ in a dense subset of $\mathcal{H}(b)$. We shall establish the equality for all $f$ in $H(\overline{\mathbf{D}})$, the space of functions holomorphic on $\overline{\mathbf{D}}$.

A few facts about Toeplitz operators are needed. It is shown in [2] that if $\psi$ is an outer function in $H^{\infty}$, then the operator $T_{\bar{\psi}}$ gives a one-to-one map of $H(\overline{\mathbf{D}})$ onto itself. This enables one to define the action of $T_{\bar{\chi}}$ on $H(\overline{\mathbf{D}})$ whenever $\chi$ is a function in the Smirnov class, that is, a ratio of two functions in $H^{\infty}$, with an outer denominator. Namely, if $\chi=\phi / \psi$ with $\phi$ and $\psi$ in $H^{\infty}$ and $\psi$ outer, and if $f$ is in $H(\overline{\mathbf{D}})$, then, as noted above, there is a unique function $g$ in $H(\overline{\mathbf{D}})$ such that $f=T_{\bar{\psi}} g$. One then defines $T_{\bar{\chi}} f$ to be $T_{\bar{\phi}} g$. It is easily seen that this definition makes sense, that is, it does not depend on the representation of $\chi$ as a ratio of $H^{\infty}$ functions. Moreover, one easily checks that if $\chi_{1}$ and $\chi_{2}$ are two functions in the Smirnov class, then $T_{\bar{\chi}_{1}} T_{\bar{\chi}_{2}} f=T_{\bar{\chi}_{1} \bar{\chi}_{2}} f$.

Let $f$ be a function in $H(\overline{\mathbf{D}})$. In showing that $\left\|\left(C_{r} f\right)^{+}\right\|_{2} \leq\left\|f^{+}\right\|_{2}$ we may assume without loss of generality that $f(0)=0$ (since, because $b(0)=0$, we do not change $f^{+}$if we subtract a constant from $f$ ). With this assumed we can write $f=z f_{0}$ with $f_{0}$ in $H(\overline{\mathbf{D}})$. We also have $b=z \beta$, with $\beta(z)=\left(1-w_{0}\right) /\left(1-w_{0} z\right)$, an invertible function in $H^{\infty}$. 
From the definition of $f^{+}$we have

$$
T_{\bar{a}} f^{+}=T_{\bar{b}} f=T_{\bar{\beta}} f_{0} .
$$

Consequently $f_{0}=T_{\bar{a} / \bar{\beta}} f^{+}$and $f^{+}=T_{\bar{b} / \bar{a}} f$. Applying the last equality to $C_{r} f$ in place of $f$, we obtain

$$
\begin{aligned}
\left(C_{r} f\right)^{+} & =T_{\bar{b} / \bar{a}} C_{r} f=T_{\bar{b} / \bar{a}} r z C_{r} f_{0} \\
& =r T_{\bar{\beta} / \bar{a}} C_{r} T_{\bar{a} / \bar{\beta}} f^{+} .
\end{aligned}
$$

Thus, we need to show that

$$
r\left\|T_{\bar{\beta} / \bar{a}} C_{r} T_{\bar{a} / \bar{\beta}} f^{+}\right\|_{2} \leq\left\|f^{+}\right\|_{2} .
$$

For that it will suffice to show that

$$
r\left\|\left\langle T_{\bar{\beta} / \bar{a}} C_{r} T_{\bar{a} / \bar{\beta}} f^{+}, h\right\rangle \mid \leq\right\| f^{+}\left\|_{2}\right\| h \|_{2}
$$

for a dense set of functions $h$ in $H^{2}$. We shall do this for the dense set $T_{a} H^{2}$. Let $g$ be in $H^{2}$ and let $h=T_{a} g$. We have

$$
\begin{aligned}
\left\langle T_{\bar{\beta} / \bar{a}} C_{r} T_{\bar{a} / \bar{\beta}} f^{+}, h\right\rangle & =\left\langle T_{\bar{\beta} / \bar{a}} C_{r} T_{\bar{a} / \bar{\beta}} f^{+}, T_{a} g\right\rangle \\
& =\left\langle T_{\bar{\beta}} C_{r} T_{\bar{a} / \bar{\beta}} f^{+}, g\right\rangle \\
& =\left\langle f^{+}, T_{a / \beta} C_{r} T_{\beta} g\right\rangle \\
& =\left\langle f^{+}, T_{a / \beta} \beta_{r} g_{r}\right\rangle \\
& =\left\langle f^{+}, T_{a / \beta} \frac{\beta_{r} h_{r}}{a_{r}}\right\rangle \\
& =\left\langle f^{+}, T_{a \beta_{r} / \beta a_{r}} C_{r} h\right\rangle .
\end{aligned}
$$

Since $C_{r}$ has unit norm as an operator on $H^{2}$, it only remains to show that $r\left\|a \beta_{r} / \beta a_{r}\right\|_{\infty} \leq 1$. We have

$$
\frac{a(z) \beta_{r}(z)}{\beta(z) a_{r}(z)}=\frac{1-z}{1-r z}
$$

Elementary reasoning shows that this function attains its maximum modulus over the unit disk at the point $z=-1$. We thus have

$$
r\left\|\frac{a \beta_{r}}{\beta a_{r}}\right\|_{\infty}=\frac{2 r}{1+r}<1
$$

as desired. The proof of Proposition 3 is complete.

Two consequences of the proposition are worth mentioning.

Corollary. If $\mu$ is a finite positive measure on $\partial \mathbf{D}$ and $f$ is in $D(\mu)$, then $\left\|C_{r} f\right\|_{\mu}$ is a nondecreasing function of $r(0<r<1)$, and $\left\|f-C_{r} f\right\|_{\mu} \rightarrow 0$ as $r \rightarrow 1$. 
The first statement in the corollary is an obvious consequence of the proposition. As for the second statement, because $C_{r} f \rightarrow f$ pointwise in $\mathbf{D}$ as $r \rightarrow 1$, while the operators $C_{r}$ are uniformly bounded as operators on $D(\mu)$, it follows by a standard property of reproducing kernel Hilbert spaces that $C_{r} f \rightarrow f$ weakly in $D(\mu)$ as $r \rightarrow 1$. Consequently $\|f\|_{\mu} \leq \lim _{r \rightarrow 1}\left\|C_{r} f\right\|_{\mu}$, while the opposite inequality follows from Proposition 3. The equality $\|f\|_{\mu}=\lim _{r \rightarrow 1}\left\|C_{r} f\right\|_{\mu}$ together with weak convergence implies that $C_{r} f \rightarrow f$ in $D(\mu)$-norm as $r \rightarrow 1$.

It should be noted that the contractivity of the operators $C_{r}$ is not a general property of de Branges-Rovnyak spaces. In fact, if $b$ is a function in the unit ball of $H^{\infty}$ such that $1-|b|^{2}$ is not log-integrable on $\partial \mathbf{D}$, then the operators $C_{r}$ do not even map $\mathcal{H}(b)$ into itself [7]. If $1-|b|^{2}$ is log-integrable then they do map $\mathcal{H}(b)$ into itself, but it can happen that they are not uniformly bounded. The author will present an example of this on another occasion.

\section{REFERENCES}

1. L. de Branges and J. Rovnyak, Square Summable Power Series, Holt, Rinehart, and Winston, New York, 1966. MR 35:5909

2. B. A. Lotto and D. Sarason, Multipliers of de Branges-Rovnyak spaces, Indiana Univ. Math. J. 42 (1993), 907-920. MR 95a:46039

3. S. Richter, A representation theorem for cyclic analytic two-isometries, Trans. Amer. Math. Soc. 328 (1991), 325-349. MR 92e: 47052

4. S. Richter and C. Sundberg, A formula for the local Dirichlet integral, Michigan Math. J. 38 (1991), 355-379. MR 92i:47035

5. S. Richter and C. Sundberg, Multipliers and invariant subspaces in the Dirichlet space, J. Operator Theory 28 (1992), 167-186. MR 95e:47007

6. S. Richter and C. Sundberg, Invariant subspaces of the Dirichlet shift and pseudocontinuations, Trans. Amer. Math. Soc. 341 (1994), 863-879. MR 94d:47026

7. D. Sarason, Sub-Hardy Hilbert Spaces in the Unit Disk, Wiley, New York, 1995.

8. D. Sarason, Harmonically weighted Dirichlet spaces associated with finitely atomic measures, in preparation.

Department of Mathematics, University of California, Berkeley, California 94720

E-mail address: sarason@math.berkeley.edu 